\title{
Obscured Galactic Giant HII Regions; Discovery of Young Clusters
}

\author{
Peter S. Conti \\ JILA and APS Department, University of Colorado, Boulder CO 80309
}

Robert D. Blum

Cerro Tololo Interamerican Observatory, La Serena, Chile

\section{Near IR Observations}

Giant HII (GHII) regions in our Galaxy are typically initially found by radio observations of their optically thin free-free continuum emission. Most of them are partially or totally obscured in the visible by the absorbing effect of intervening and/or local interstellar dust. We (Blum et al. 1999, 2000) have selected a list of the brightest GHII regions in our Galaxy (from Smith et al. 1978) and have begun a program of $J H K$ imaging and $K$ band spectroscopy to identify and classify the exciting stars. We have obtained near IR imaging of eight GHII regions (and data is available for four others). All of these, aside from W49, show the presence of a stellar cluster in the $K$ band at the radio source position. The $K, H-K$ diagrams are used to select the brightest stars. The $J-K$ vs. $H-K$ diagrams distinguish those stars along the normal reddening line from those with $K$ band excesses. The former group ought to have normal OB star spectra; the latter will have featureless continua in the $K$ band due to emission from localized warm dust arising in a natal disc (Hanson et al. 1997). A disc geometry can also produce $\mathrm{CO}$ emission (or absorption) band features.

\section{Classifications of the Brightest Cluster Stars}

As a first step in classification, we have obtained $K$ band spectra of a few of the brightest stars from the C-M diagram for most of the clusters. In Table 1 , we list the 12 GHII regions with remarks about the spectra of the brightest stars, from ongoing work with A. Damineli (aside from M17 from Hanson et al. 1997, NGC3603 from Drissen et al. 1995, and W51 from Hanson - private communication). This sample represents about half of the brightest GHII listed by Smith et al. 1978. Curiously, at least five of the clusters (out of eight with spectra available) contain one or more stars with evidence of stellar discs from featureless $K$ band continuua, $K$ band excesses, and/or CO emission lines. While this may only be small (source) number statistics, it does suggest that either the natal disc phase for massive stars lasts an appreciable fraction of the OB star lifetime or radio selected GHII regions are not sampled uniformly over the massive star main sequence lifetime. 
Table 1. Bright Galactic GHII with Imaging Data

\begin{tabular}{c|c|c|c|l}
\hline $\begin{array}{c}\text { Catalog } \\
\text { Name }\end{array}$ & $\begin{array}{c}\text { Radio } \\
\text { Source }\end{array}$ & $\begin{array}{c}\text { Dist. } \\
\text { Kpc }\end{array}$ & $\begin{array}{c}\text { \# Lyc } \\
\times 10^{49}\end{array}$ & $\begin{array}{l}\text { Remarks on Individual } \\
\text { Stellar Spectra }\end{array}$ \\
\hline NGC3576 & G291.3-0.7 & 3.6 & 26 & CO emiss + CO abs + no lines \\
NGC3603 & G291.6-0.5 & 8.2 & 188 & several Of/WN + several early O \\
- & G333.1-0.4 & 13.4 & 169 & Cluster; No spectra yet \\
- & G333.6-0.2 & 14.1 & 1140 & 2 (?) Clusters; No spectra yet \\
- & G351.6-1.3 & 4.4 & 11 & LBV + CO abs + no lines \\
W31 & G10.2-0.3 & $5.1:$ & 30 & 1 no lines + 4 early O \\
W33 & G12.8-0.2 & 4.6 & 5 & 1 disc (Br $\gamma$ emission) \\
M17 & G15.1-0.7 & 2.3 & 54 & OB stars; some with discs \\
W42 & G25.4-0.2 & 13.4 & 82 & 1 early O + 2 no lines \\
W43 & G30.8-0.0 & 7.0 & 107 & Of/WN + 2 early O \\
W49 & G43.2-0.0 & 13.8 & 172 & YSOs ? \\
W51 & G49.5-0.4 & 6.6 & 154 & 1 early O (others S/N problem?)
\end{tabular}

\section{The Special Case of W49}

There are about 30 point-like radio sources visible in the core of $\mathrm{W} 49$ at $\mathrm{cm}$ wavelengths (De Pree et al. 1997). They interpret these optically thick free-free sources to be individual ultra compact HII (UCHII) regions (Wood \& Churchwell 1989). W49 is thus a massive $O B$ star cluster. In most cases, there is no stellar image in the $K$ band corresponding to the radio point source position. This implies that the exciting stars are deeply embedded in their natal clouds, composed of extensive dust that absorbs in the near IR. This interpretation has recently been strengthened by the detection of re-radiated dust emission from many of the radio point sources in the thermal IR by Smith et al. (2000). All the other GHII regions in our Galaxy that we have observed have a stellar cluster visible in the $K$ band, whereas in W49 the cluster is buried in its birth material. W49 is the youngest example of a cluster of newly born massive stars in our Galaxy and intermediate in luminosity between UCHII regions and the buried SSC in starburst galaxies that are the subject of the next two talks.

\section{References}

Blum, R.D., Conti, P.S., \& Damineli, A. 1999, AJ, 117, 1392

Blum, R.D., Conti, P.S., \& Damineli, A. 2000, AJ 119, 1860

De Pree, C.G., Mehringer, D.M., \& Goss, W.M. 1997, ApJ, 482, 307

Drissen, L., Moffat, A.F.J., Walborn, N., \& Shara, M.M. 1995, AJ, 110, 2235

Hanson, M.M., Howarth, I.D., \& Conti, P.S. 1997, ApJ, 489, 698

Smith, L.F., Biermann, P., \& Mezger, P.G. 1978, AA, 66, 65

Smith, N., Jackson, J.M., Kraemer, K.E., Deutsch, L.K., Bolatto, A., Hora, J.L., Fazio, G., Hoffman, W.F., \& Dayal, A. 2000, ApJ, 540, 316

Wood, D.O.S., \& Churchwell, E. 1989, ApJ Suppl, 69, 831 